\title{
Vilnius declaration
}

\author{
IFIP World Information Technology Forum (WITFOR) \\ International Federation for Information Processing, Hofstrasse 3, A 2361 Laxenburg, \\ Austria, Tel: +43223673616 , Fax: +432236736169
}

ifip@ifip.or.at; www.ifip.or.at

Abstract: Participants from 68 countries at The First World Information Technology Forum (WITFOR), organised by IFIP under the auspices of UNESCO and hosted by the Government of Lithuania, gathered in Vilnius, Lithuania, 27-29 August 2003. They addressed the following major goals: Bridging the digital divide; Ensuring the freedom of expression; Reducing poverty through the use of education and Information and Communications Technology (ICT); Facilitating social integration; Respecting linguistic and cultural diversity; Fostering the creation of public domains; Supporting communities in fighting illiteracy; Encouraging e-governance and e-democracy initiatives; Improving the quality of life; and Protecting the local and global environment. The Forum conducted its work through 8 commissions: Preparing the ground for ICT; Building the infrastructure; Economic opportunities; Empowerment and participation; Health; Education; Environment; and Social and ethical aspects of the Information Society.

Key words: cultural diversity, Digital Divide, e-democracy, e-governance, environment, freedom of expression, illiteracy, linguistic diversity, poverty, public domain, quality of life, social integration 


\section{INTRODUCTION}

Participants from 68 countries at The First World Information Technology Forum (WITFOR), organised by IFIP under the auspices of UNESCO and hosted by the Government of Lithuania, gathered in Vilnius, Lithuania, 27-29 August 2003, have addressed through the Forum the following major goals:

- Bridging the digital divide between rich and poor in the world; urban and rural societies; men and women; and different generations;

- Ensuring the freedom of expression enshrined in Article 19 of the universal declaration of human rights and other such instruments;

- Reducing poverty through the use of education and Information and Communications Technology (ICT);

- Facilitating the social integration of excluded segments of societies;

- Respecting linguistic and cultural diversity;

- Fostering the creation of public domains with full respect of intellectual property rights;

- Supporting communities in fighting illiteracy;

- Encouraging e-governance and e-democracy initiatives;

- Improving the quality of life through effective health service systems;

- Protecting the local and global environment for future generations.

\section{CONTEXT}

We as participants are:

- Aware of the complexity facing national governments in developing reliable and affordable ICT;

- Further aware of the importance and need of safe and secure ICTs as the foundation of global, regional and local Information and Communication services, supporting governments, organizations, enterprises and individuals;

- Convinced that governments need to build upon ICT- related achievements and independently evaluate existing pilot projects from the perspective of beneficiaries;

- Subscribing to the importance of safeguarding the economic, social, environmental and cultural rights of all peoples, with special attention to the protection of traditional societies and indigenous people;

- Believing in the equitable and ethical sharing of the benefits of ICT and the minimisation of any negative impacts; 
- Fully accepting the realities facing often demanding partners, especially in the sector of economic investment required to set up the physical infrastructure;

- Conscious that most of the discussions on the future of the information society is being driven by technology push more than by citizens' needs

\section{DECLARATION}

We call upon national governments, civil societies and other partners to commit themselves to the implementation of the above stated objectives and to translate their commitment to the development of ICT through the creation of a favourable environment for partnership and economic investment. We resolve to work closely with all the above-mentioned partners and commit ourselves to the following strategic actions:

- Inviting national governments to give priorities to national socioeconomic development plans for the creation of ICT infrastructures through:

- International co-operation among central governments and through international development agencies;

- The establishment of public and private partnerships as the cornerstone of the deployment of ICT at the local and national levels;

- Facilitating investments in the physical infrastructure by international and regional financial institutions;

- Supporting the development of new ICT tools and contributing to international programmes for ICT advancement;

- Ensuring affordable and equitable accessibility to ICT between urban and rural communities and between men and women, taking into consideration the existing generation gap.

- Urging national governments to guarantee the application of the principles of freedom of expression and privacy through appropriate legislation that will:

- Implement these principles as they apply to traditional media, also to the Internet, and satellite broadcast;

- Promote public access to data and information of public interest which is held by governments, private organizations or companies.

- Ensuring a continuous process of education on the rights of citizens as a fundamental element of poverty alleviation by: 
- Facilitating affordable universal access to the Internet and encouraging networking and dialogue between the diverse communities of interest.

- Facilitating knowledge and information sharing (especially as it affects the rights of the poor and the excluded) and facilitating their progressive integration into the fabric of cities, towns and societies to reduce existing social tensions and conflicts.

- Encouraging international cooperation for the provision of safe and secure information and communication networks and systems.

- Supporting the development and adoption of free and Open Source solutions wherever more affordable and /or suitable than proprietary solutions.

- Promoting a harmonious society within the cultural diversity of countries, convinced that national languages must never be seen as an obstacle to access to ICT.

- Facilitating an environment and a physical and legal infrastructure for the establishment of public domains where:

- Universal access to content is guaranteed as an essential part of the freedom of expression with due respect to legislation governing the rights to intellectual property.

- Empowering all communities, especially grass roots communities, through systematic programmes aimed at developing literacy, including ICT literacy, which progressively involve community members in cooperative actions.

- Encouraging the use of new ICT tools, especially with regard to the new development paradigm in e-governance and e-democracy:

- Giving due regard to social and ethical aspects and the special needs of different groups in society;

- Empowering them to benefit from the digital revolution.

- Promoting the use of ICTs to address the basic needs of communities, particularly by creating a modern social health system that would improve their quality of life with special emphasis on: 
- Targeting major health problems in developing countries notably HIV/AIDS, TB, Malaria and mother and child health care, through effective health management information systems;

- Optimizing the use of free and open source software, models and component specifications in future health information systems;

- Intensifying training and education in local adaptation, maintenance and use of health related information systems.

- Improving the use and application of ICTs in projects aimed at protecting the local and global environment for future generations, and in developing systems for monitoring potentially environment-threatening process and systems that will ensure a continued healthy environment.

We, the participants, representing national governments, business communities, NGOs, IGOs, academia and international organisations invite all partners to translate the above strategic actions into implementable action plans. We call upon all national, regional and international financial institutions to be involved in the implementation of these action plans by investing in the necessary development of ICTs at local, regional and national levels.

\section{BACKGROUND}

The Forum conducted its work through 8 commissions:

1. Commission 1 Preparing the ground for ICT;

2. Commission 2 Building the infrastructure;

3. Commission 3 Economic opportunities;

4. Commission 4 Empowerment and participation;

5. Commission 5 Health;

6. Commission 6 Education;

7. Commission 7 Environment;

8. Commission 8 Social and ethical aspects of the information society.

Below follows a summary of their findings.

\section{Preparing the ground for ICT}

Availability and use of ICTs across a spectrum of public and business domains is rightly highlighted as a crucial area of government action for development. To date, however, there has been a depressingly low rate of 
success in such efforts, largely due to an overly technocratic approach to the problem. ICT policies for the diffusion of technology must be made in the context of development priorities and be accompanied by actions to create socio-economic conditions that enable local communities to appropriate ICTs for the improvement of their lives. To prepare for ICT and successful participation in the Information Society, there needs to be sustained government action in understanding and responding to social demand, reshaping national education, building indigenous science/technology/engineering capabilities, and effecting economic and social reform.

Understanding and responding to social demand includes creating awareness regarding opportunities offered by ICT for socio-economic change, as well as the effort involved in such change; use of ICT in government organisations; interventions to empower socially excluded groups.

Reshaping national education includes tackling illiteracy; development of computer and information skills; professional engineering and management capabilities; development of critical abilities.

Building indigenous science/technology/engineering capabilities includes cultivating a socio-economic context of innovation; tertiary education curricula for engineering and management; local R\&D balancing export opportunities and local needs, international R\&D.

Effecting economic and social reform includes measures for appropriate economic liberalization and the development of an effective market socioeconomic regime; support of entrepreneurial activities, social policies to alleviate the destructive effects of socio-economic innovation; legal frameworks for the information economy; political mechanisms enabling citizens to participate in local and global socio-economic change and negotiate their preferred life conditions.

\section{Building the infrastructure}

There are three themes for ICT development:

1. Connectivity to the communications infrastructure;

2. Capacity to make effective use of ICTs;

3. Content of information and knowledge stored on and transmitted by the ICT networks. 
Infrastructure should not be considered as only equipment, but should be understood as: Technology, Services, Human resources, Legal and regulatory frameworks and Economy. Some recommended actions for building a sustainable ICT infrastructure are:

- Adherence to International standards should be encouraged and when appropriate enforced.

- Operation and maintenance should be considered from the very beginning of any ICT project to ensure project success and sustainability. "Do not invest in what you cannot maintain".

- Promotion of universal Internet access should be supported to minimize the risk of widening the digital divide.

- Implementation of new ICT services should be encouraged.

- On-going education at three different levels: executive awareness level,

- user skills level and professional technical level.

- Governments should formulate a long-term vision, set policies, regulate, protect the users and control the quality of services.

- Regulatory authorities should be in charge of a comprehensive regulatory framework to enforce efficiency, competition, transparency and universal access.

\section{Economic opportunities}

Developed and developing countries are divided by a multitude of economic, social, cultural and other issues, but arguably the most significant divide at present is digital. Combined with the globalization of trade, new digital technologies are presenting economic opportunities and creating wealth at a rate that threatens to increase the divide. The level of developing countries' involvement and use of ICT slowly improves but many countries are unable to make real progress in economic development without assistance.

A comprehensive set of national policies and strategies prepared by WITFOR's Economic Opportunity Commission provides clear paths to bridge the digital divide. Timing is a key factor. If developing countries are delayed in their digital development then the rapid growth of the developed world may leave them impossibly far behind. 


\section{Empowerment and participation}

With ICT governments is able to improve the quality and expand the reach and accessibility of the services they offer to their citizens. In that way good governance is the goal and e-Government is the way. Building up modern governance in the information society the approach has to be citizencentred, cooperative, seamless and polycentric.

There are four postulates that have to be stated for achieving good practice (according to the 2003 eEurope Awards Conference):

- e-Government is the key to good governance in the information society;

- e-Government is impossible without having a vision;

- e-Government is not just about technology but about a change in culture;

- e-Government is not just about service delivery but about a way of life.

Up to now the low take up of public e-services has been a serious problem. To improve this situation, some conditions have to be met:

- Services must become less bureaucratic and citizens have to get economic and individual benefits.

- The needs of specific target groups must be addressed.

- A multi-channel access mix is necessary with a diversity of contact points: home and mobile, kiosk, citizen office and multifunctional service shops.

- A single-window access must be provided for all services regardless of government level and agency.

- Knowledge enhancement must be interwoven into service processes.

- Special promotions must concentrate on individual groups of addressees: rural and traditionally underserved communities; the younger.

- Democratic decision-making has to stress citizen participation. For this the interaction between individuals and organizations has to be sustained by electronic services.

\section{Health}

IT strategies in health care should target the major health problems in developing countries, such as HIV/AIDS, TB, Malaria and mother and child health. Developing countries should therefore prioritise Health Management Information Systems, using multiple sources of aggregated and anonymised data from different related sectors in society, aiming at strengthening health management and primary health care delivery including a basic hospital structure. Integration within and between health care establishments requires the specification of data sets and terminology to be consistent. 
Future health information systems should optimally use free and Open Source Software, models and component specifications characterised by: scalability and flexibility through a component-based architecture enabling free combination of relevant services allowing for incremental development; portability separating logical and technological specifications; and a finegrained architecture to reduce complexity.

Sustainable systems must be based on: training and institutional development enabling local adaptation, maintenance and use; leadership of health professionals and other domain experts in systems development; and must focus on the local use of information for action.

\section{Education}

\section{Nature of education}

The nature of education is to improve a person's relation to theworld. The organization, methods, structures and objectives of education should be brought into alignment with the knowledge society.

\section{Lifelong learning}

ICT can allow education to be spread around many communities, and promote lifelong learning and capacity building for the whole community.

\section{E-inclusion}

ICT should be used to reduce the education inequalities. Women, unemployed and disadvantaged people (refugees, disabled, etc.) should receive special attention in this process. National contents must be developed in locallanguages.

\section{Computer literacy}

At all levels of education, computer literacy and ICT competence for the knowledge society should be achieved, adapted to local conditions.

\section{Teacher education}

Any educational system reform should start with teacher in-service and pre-service education. Teachers should be encouraged to acquire and use ICT equipment and skills. 


\section{Environment}

Need for identification and capacity building

- Identification of special needs of Least Developed Countries (LDCs) and indigenous people, safeguarding of their local culture.

- Capacity building efforts leading to self-sufficiency in all aspects of training and supporting programs and technologies in ICT for the environment.

\section{Public policy and access}

- Development and implementation of equitable strategies for ICT, including access to affordable systems and connectivity.

- Decreasing non-tariff barriers on ICT and the environment.

- Promotion and establishment of policy alternatives that provide for equitable growth and development.

- Promotion and establishment of policies that promote the links between

- environmental knowledge, data quality and human health, environmental degradation, natural disasters, climate change, food security, water supply and quality, and other related issues.

- Improvement of public accessibility and understanding of ICT and environmental issues. This includes the roles of public data access and data sharing between organizations, regions and countries.

\section{Monitoring and regulatory issues}

- Identification, development and promotion of the role of ICT in environmental monitoring processes and the regulation of environmental issues. This includes local or regional monitoring for regulatory processes and monitoring as part of regional or global networks (for example, global climate change monitoring networks).

- Establishment of networks for monitoring that provide equitable access to facilities, equipment, training and communications so that there is both data sharing and technology transfer.

\section{Social and ethical aspects of the Information Society}

Most of the discussions on the future of the information society suggest that it is being determined by technical feasibility and driven by technology 
push more than by users' and customers' needs. Little attention is paid to social impact and ethics - except in the field of education and culture.

Among the social and ethical concerns we strongly suggest a focus on professional ethics; access to content and technology for all; education, literacy and public awareness; multilingualism, cultural concerns; influence of globalization; regulation, self-regulation, governance and democratic participation; intellectual property rights; specific digital policies such as eHealth, eWork, eGovernment, etc; privacy; protection of human and civil rights; protection of the individual against surveillance; development of the quality of life and well-being; combating social exclusion; computer crime, cyber-attacks and security; employment and participative design at work; risk and vulnerabilities.

It is recommended to establish national or regional social and ethical committees to assess these issues and develop social and ethical benchmarks, to ensure that the balance between technical and social aspects is maintained. If we are to enter an information and knowledge society we must remain critical and human. 